Published in final edited form as:

Nat Neurosci. 2018 January ; 21(1): 29-32. doi:10.1038/s41593-017-0023-y.

\title{
Accumbal D2 cells orchestrate innate risk-avoidance according to orexin signals
}

\author{
Craig Blomeley", Celia Garau\#, and Denis Burdakov \\ The Francis Crick Institute, London NW1 1AT \\ \# These authors contributed equally to this work.
}

\begin{abstract}
Excitation of accumbal D2 cells governs vital actions including avoidance of learned risks, but the origins of this excitation and roles of D2 cells in innate risk-avoidance are unclear. Hypothalamic neurons producing orexins/hypocretins enhance innate risk-avoidance via poorly understood neurocircuits. We describe a direct orexin $\rightarrow \mathrm{D} 2$ excitatory circuit, and show that D2 cell activity is necessary for orexin-dependent innate risk-avoidance in mice, thus revealing an unsuspected hypothalamus-accumbens interplay in action selection.
\end{abstract}

Innate ability to avoid lethal risks is key for survival in all organisms. In mammals, contextappropriate actions such as risk-avoidance are computed by the brain, but relations between the underlying neural signals are incompletely understood. Orexin/hypocretin peptides are a fundamental brain system required for context-appropriate brain state control1, 2. They are made exclusively by a subset of lateral hypothalamic (LH) neurons, which are activated by diverse internal and external stresses, and evoke central and autonomic arousal by releasing orexin at brain-wide projections1, 2, 3, 4. Previous studies indicated that the orexin signals are sufficient and necessary for inducing anxiety-like states, such as increased innate riskavoidance (a hallmark of anxiety5) in rodents, or panic attacks in humans6, 7, 8, 9. However, it remains unknown what downstream circuits are essential for innate risk-avoidance driven by natural orexin signals. Among brain areas innervated by orexin ${ }_{\mathrm{LH}}$ cells is the nucleus accumbens (NAc)4, a central switch of action strategies10. The NAc contains dopamineinhibited D2 NAc $_{\text {nc }}$ neurons11 (Fig. S1A,B), whose excitation was recently found to drive avoidance of learned risk10. However, it is unclear where the D2 $\mathrm{NAc}_{\mathrm{c}}$ cell excitation physiologically originates, and whether it drives innate risk-avoidance.

To test whether $\mathrm{D} 2_{\mathrm{NAc}}$ cell excitation may originate from orexin $\mathrm{LH}_{\mathrm{LH}}$ neurons, we optogenetically photo-stimulated NAc orexin axons, or applied orexin peptide, while

Corresponding author: Prof. Denis Burdakov, d.burdakov@oxon.org.

Accession Codes: $\mathrm{n} / \mathrm{a}$

Data Availability Statement: The data that support the findings of this study are available from the corresponding author upon reasonable request

Author Contributions: CB and CG (equal contributions) designed and performed experiments and analysed data; DB designed experiments, obtained funding, and wrote the paper.

Competing Financial Interest

The authors have no competing interests. 
recording from $\mathrm{D} 2_{\mathrm{NAc}}$ cells in mouse brain slices (Fig. 1A-D; see Supplementary Methods). The orexin stimulations excited D2 ${ }_{\mathrm{NAc}}$ cells on a time-scale similar to the risk-avoidanceassociated $\mathrm{D} 2_{\mathrm{NAc}}$ cell excitation in vivo10, and this excitation was abolished by orexin receptor antagonism (Fig. 1D,E; a low-probability fast orexin cell $\rightarrow$ D2 cell excitation was also present in some cells, Fig. S1F). Orexin stimulation did not affect most other NAc cells, such as NAc D1 cells (Fig. 1E and Fig. S2A,B; this suggests that D1 and D2 cells studied here were largely non-overlapping as also found in 12), or NAc fast-spiking or ACh interneurons ( $\mathrm{n}=12$ and 10, respectively, data not shown). However, orexin stimulation excited NPY ${ }_{\text {NAc }}$ interneurons (Fig. 1E, Fig. S1C-E), which according to previous work may indirectly stimulate $\mathrm{D} 2_{\mathrm{NAc}}$ cells by inhibiting $\mathrm{D} 1_{\mathrm{NAc}}$ cells 13 . The $\mathrm{LH}$ contains melaninconcentrating hormone $(\mathrm{MCH})$ neurons that are distinct from orexin neurons4. We found, by rabies-assisted retrograde tracing of direct inputs to $\mathrm{D} 2_{\mathrm{NAc}}$ cells, that $\mathrm{D} 2_{\mathrm{NAc}}$-projecting $\mathrm{LH}$ cells are more likely to contain orexin than $\mathrm{MCH}$ (Fig. 2); and optogenetic stimulation of NAc MCH axons did not change D2 ${ }_{\mathrm{NAc}}$ cell excitability (Fig. S2C,D). Together, these data demonstrate cell-type-specific $\mathrm{LH} \rightarrow \mathrm{NAc}$ circuitry that would be expected to directly and indirectly increase $\mathrm{D} 2_{\mathrm{NAc}}$ cell activity.

To probe the roles of natural orexin $\mathrm{LH}_{\mathrm{H}}$ and $\mathrm{D} 2_{\mathrm{NAc}}$ signals in action selection, we quantified the intensity of innate risk-avoidance behavior in two assays (open space and predator odor avoidance, see Supplementary Methods), combined with chemogenetic and pharmacological modulation of D2 ${ }_{\mathrm{NAc}}$ and orexin signals. Our main aim was to examine behavioral roles of natural (i.e. spontaneously-generated by the brain) orexin and $\mathrm{D} 2_{\mathrm{NAc}}$ cell signals, which we isolated by comparing behavior resulting from natural vs. signal-specifically suppressed brain function. Suppression of natural orexin signaling by orexin receptor antagonism (Fig. 3B-E) decreased risk-avoidance behaviors (left plots in Figs. 3C and E, relevant statistics are shown in green; importantly, these effects were not associated with locomotor sedation, Fig. $\mathrm{S} 4 \mathrm{E})$. Thus, orexin is necessary as well as sufficient6, 7, 9 for normal innate risk-avoidance (the sufficiency of NAc orexin stimulation for risk-avoidance was further confirmed in our behavioral assays, Fig. S3). By chemogenetically silencing or stimulating $\mathrm{D} 2_{\mathrm{NAc}}$ cells (Figs. $3 \mathrm{~A}$ and $\mathrm{S} 4 \mathrm{~A}, \mathrm{~B}$ ), we also found that $\mathrm{D} 2_{\mathrm{NAc}}$ cells were necessary (left plots in Figs. $3 \mathrm{C}$ and $\mathrm{E}$, relevant statistics are shown in purple) and sufficient (Fig. S4A-D) for normal innate riskavoidance.

We next examined co-dependencies of risk-avoidance arising from natural orexin or D2 $\mathrm{NAc}$ signals, by combinatorial silencing of global orexin receptors and local $\mathrm{D} 2_{\mathrm{NAc}}$ cells. We found that risk-avoidance driven by natural orexin signals (isolated by quantifying behavior with and without orexin antagonist in individual mice) was abolished by $\mathrm{D} 2_{\mathrm{NAc}}$ cell silencing (Fig. 3C,E: center plots). This indicates that translation of natural orexin signals into innate risk-avoidance requires $\mathrm{D} 2_{\mathrm{NAc}}$ cells, and that other orexin-excited cells1, including NPY $\mathrm{NAc}_{\mathrm{c}}$ cells identified here, are not sufficient for this translation. Finally, we analyzed whether natural orexin signals are necessary for the behavioral output D2 ${ }_{\mathrm{NAc}}$ cells. We found that risk-avoidance driven by natural D2 ${ }_{\mathrm{NAc}}$ cell activity (isolated by quantifying behavior with and without $\mathrm{CNO}$ in individual $\mathrm{D} 2 \mathrm{NAc}_{\mathrm{N}}-\mathrm{hM} 4 \mathrm{Di}$ mice) was substantially reduced by orexin receptor blockade (Fig. 3C,E: left plots), suggesting that $\mathrm{D} 2_{\mathrm{NAc}}$ cells regulate behavior according to orexin tone. Thus, orexin is not only sufficient to stimulate 
$\mathrm{D} 2_{\mathrm{NAc}}$ cells (Fig. 1D), but also necessary for driving their behaviorally-relevant output in vivo (Fig. 3C,E).

Overall, these findings reveal an excitatory drive to $\mathrm{D} 2_{\mathrm{NAc}}$ cells that emanates from $\mathrm{LH}$ orexin cells, and show that $\mathrm{D} 2_{\mathrm{NAc}}$ cells are essential for innate risk-avoidance mediated by spontaneously-released orexin. These findings suggest interesting hypotheses for further study. For example, orexin cell activity is implicated in many types of reward-seeking 14,15, and it is tempting to speculate that the enhanced risk-avoidance caused by orexin may help avoid danger while seeking rewards. Since dopamine inhibits but orexin excites D2 ${ }_{\mathrm{NAc}}$ cells, $\mathrm{D} 2 \mathrm{NAc}$-dependent risk-avoidance may be computed from antagonistic integration of these neurochemical representations of reward and stress. Insights into neuropsychiatric disorders linked to orexin signals6 will benefit from understanding how this LH system governs molecularly-defined brain switches of action strategies.

\section{Supplementary Material}

Refer to Web version on PubMed Central for supplementary material.

\section{Acknowledgements}

This work was funded by The Francis Crick Institute, which receives its core funding from Cancer Research UK, the UK Medical Research Council, and the Wellcome Trust.

\section{References}

1. Sakurai T. Nature Reviews Neuroscience. 2014; 15:719-731. [PubMed: 25301357]

2. Giardino WJ, de Lecea L. Current Opinion in Neurobiology. 2014; 29:103-108. [PubMed: 25050887]

3. Gonzalez JA, Iordanidou P, Strom M, Adamantidis A, Burdakov D. Nature Communications. 2016; 7:11395.

4. Peyron C, et al. The Journal of Neuroscience. 1998; 18:9996-10015. [PubMed: 9822755]

5. Maner JK, Schmidt NB. Behav Ther. 2006; 37:181-189. [PubMed: 16942970]

6. Johnson PL, et al. Nature Medicine. 2010; 16:111-115.

7. Suzuki M, Beuckmann CT, Shikata K, Ogura H, Sawai T. Brain Research. 2005; 1044:116-121. [PubMed: 15862796]

8. Bonnavion P, Jackson AC, Carter ME, de Lecea L. Nature Communications. 2015; 6:6266.

9. Heydendael W, Sengupta A, Beck S, Bhatnagar S. Physiology \& Behavior. 2014; 130:182-190. [PubMed: 24140988]

10. Zalocusky KA, et al. Nature. 2016; 531:642-646. [PubMed: 27007845]

11. Nicola SM, Surmeier J, Malenka RC. Annual Review of Neuroscience. 2000; 23:185-215.

12. Kupchik YM, et al. Nature Neuroscience. 2015; 18:1230-1232. [PubMed: 26214370]

13. Koos T, Tepper JM. Nature Neuroscience. 1999; 2:467-472. [PubMed: 10321252]

14. Harris GC, Wimmer M, Aston-Jones G. Nature. 2005; 437:556-559. [PubMed: 16100511]

15. Mahler SV, Moorman DE, Smith RJ, James MH, Aston-Jones G. Nature Neuroscience. 2014; 17:1298-1303. [PubMed: 25254979] 


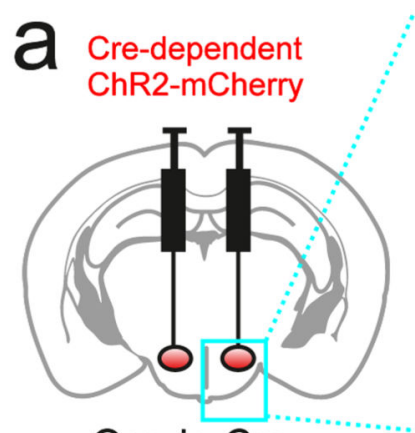

Orexin-Cre

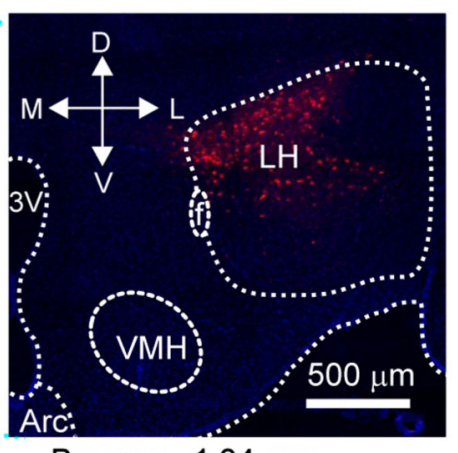

Bregma - $1.34 \mathrm{~mm}$

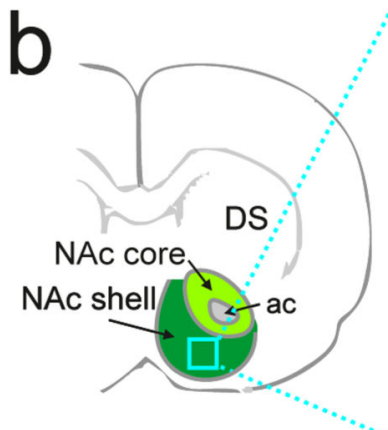

NAc core NAc shell

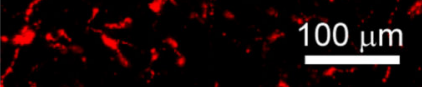

Bregma $+1.50 \mathrm{~mm}$
C

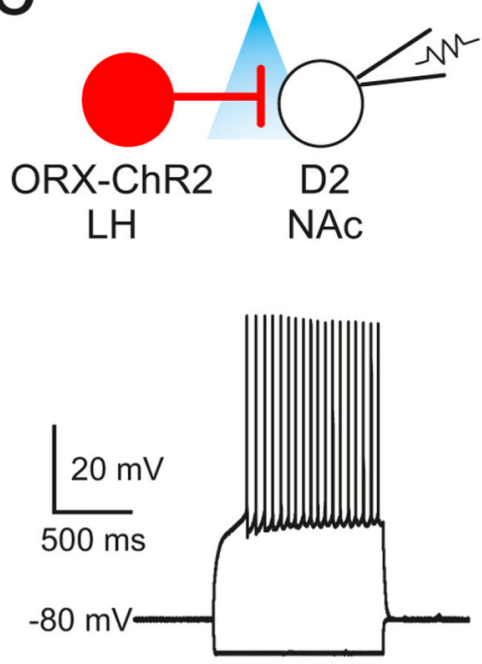

e

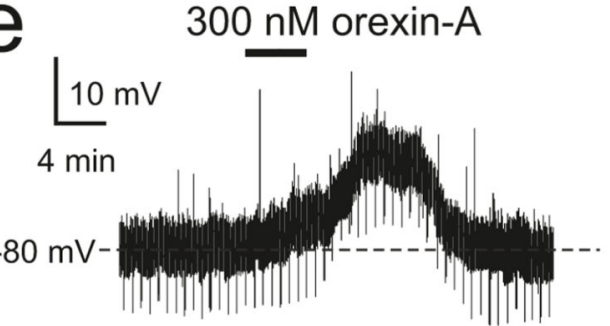

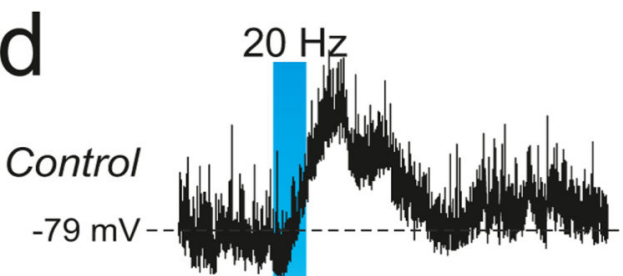
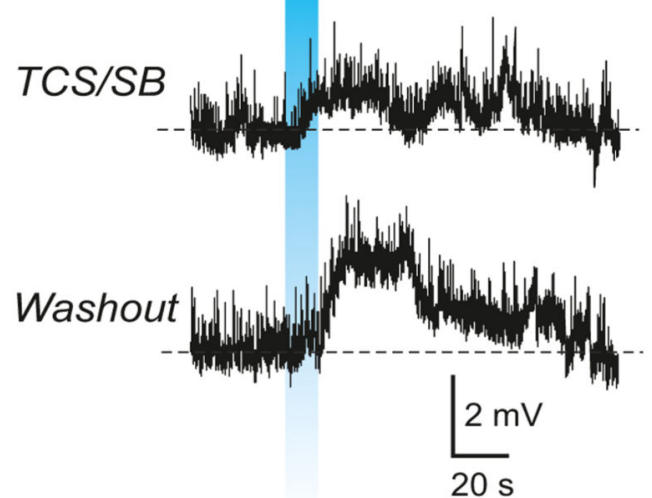

0.0002

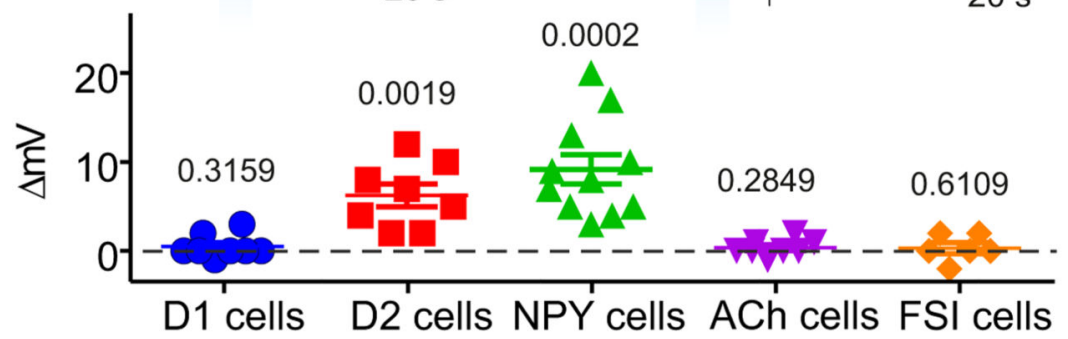

Fig. 1. Functional evidence for orexin $\mathrm{LH}_{\mathrm{H}} \rightarrow \mathrm{D} \mathbf{N}_{\mathrm{NAc}}$ excitation.

A. Left: targeting ChR2 to orexin cells. Right: expression of ChR2 in LH, representative example of 5 brains. $3 \mathrm{~V}=$ third ventricle; Arc $=$ arcuate nucleus; $\mathrm{f}=$ fornix; $\mathrm{M}, \mathrm{L}, \mathrm{D}, \mathrm{V}=$ medial, lateral, dorsal, ventral.

B. Orexin cell axons (ChR2-mCherry, red) in NAc shell, representative example of 5 brains. $\mathrm{DS}=$ dorsal striatum, $\mathrm{ac}=$ anterior commissure.

C. Top: recording scheme. Bottom: defining electrical fingerprint of a $\mathrm{D} 2_{\mathrm{NAc}}$ cell (see Supplementary Methods).

D. Top row: effect of orexin axon photo-stimulation (blue) on $\mathrm{D} 2 \mathrm{NAc}_{\mathrm{NA}}$ cell membrane potential (left) or current (right); representative example of $n=22$ cells. Lower rows: same 
experiment with orexin receptor blockade (TCS/SB, see Supplementary Methods), representative example of $n=5$ cells.

E. Left: effect of bath-applied orexin on a $\mathrm{D} 2_{\mathrm{NAc}}$ cell (representative example of $\mathrm{n}=8$ cells). Right: group data (raw values for individual cells and mean \pm sem) for different NAc cell types. Numbers above data are $\mathrm{p}$ values from one-sample two-tailed $\mathrm{t}$-tests ( $\mathrm{t}, \mathrm{df}$ from left to right $=1.08,7 ; 4.84,7 ; 5.56,10 ; 1.16,7 ; 0.5423,5)$. 

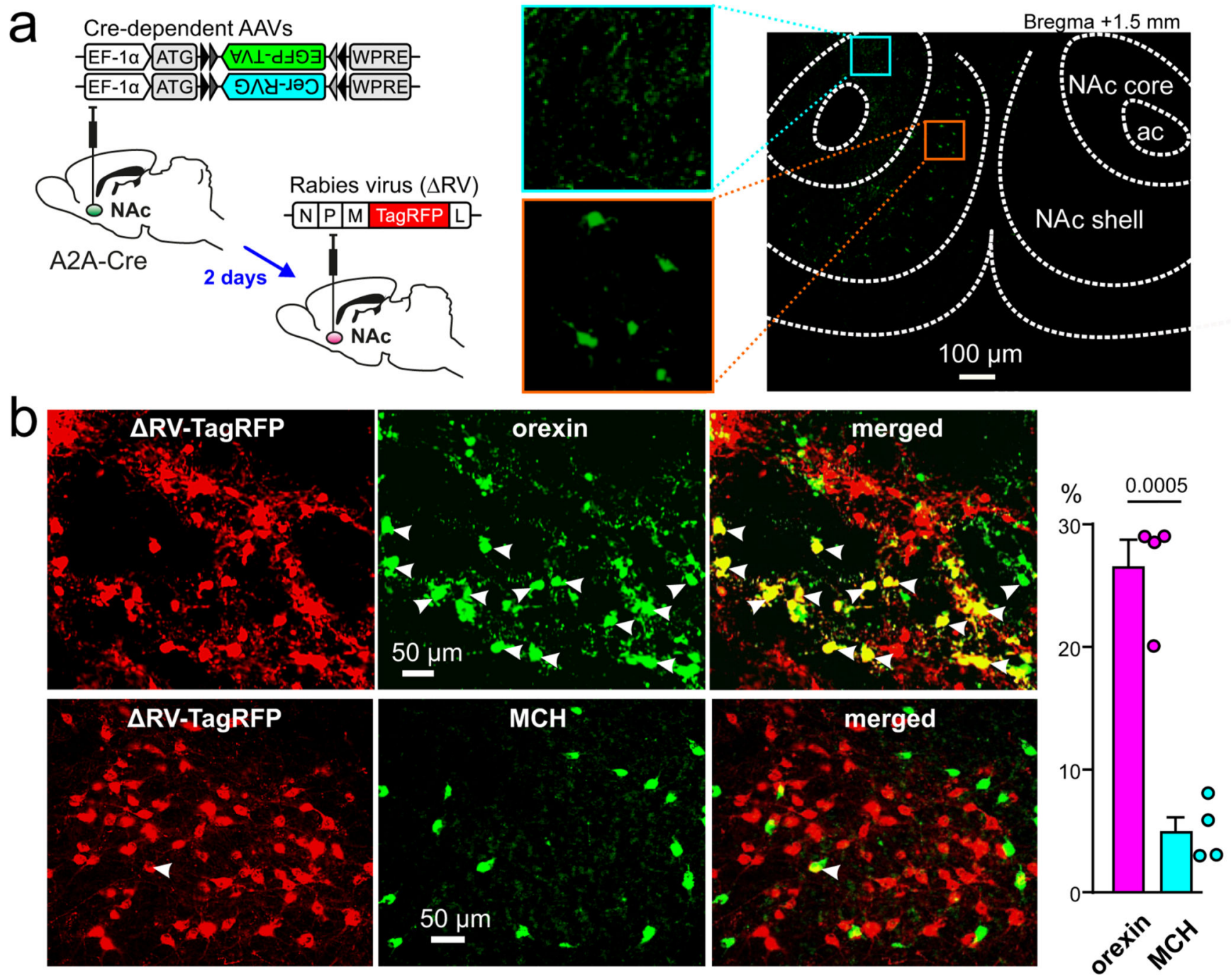

Fig. 2. Anatomical evidence for a direct $\operatorname{orexin}_{\mathbf{L H}} \rightarrow \mathbf{D} \mathbf{2}_{\mathrm{NAc}}$ circuit.

A. Left: targeting strategy. Right: eGFP expression in D2 ${ }_{\mathrm{NAc}}$ shell neurons, zoomed images are included to confirm labelling of cell bodies in NAc shell rather than core (representative example of $n=4$ brains).

B. Left histology images: Orexin and $\mathrm{MCH}$ immunoreactivity in LH neurons that directly innervate D2 ${ }_{\mathrm{NAc}}$ cells, representative examples from $\mathrm{n}=4$ brains. Right plot: incidence of orexin and $\mathrm{MCH}$ immunoreactivities $\mathrm{D} 2_{\mathrm{NAc}}$-projecting $\mathrm{LH}$ neurons (values are mean \pm sem and raw values for individual brains). Number above bars is $\mathrm{p}$ value from unpaired t-test with Welch's correction, t, df $=8.611,4.723$ (analysis of 1636 cells from 4 brains). 

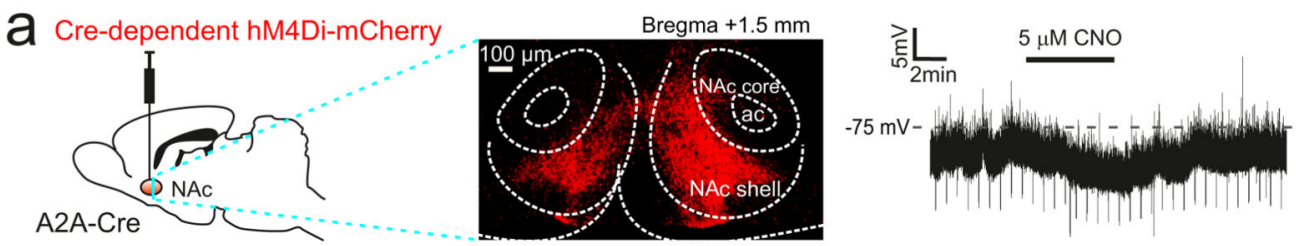

b Open field center-border exploration
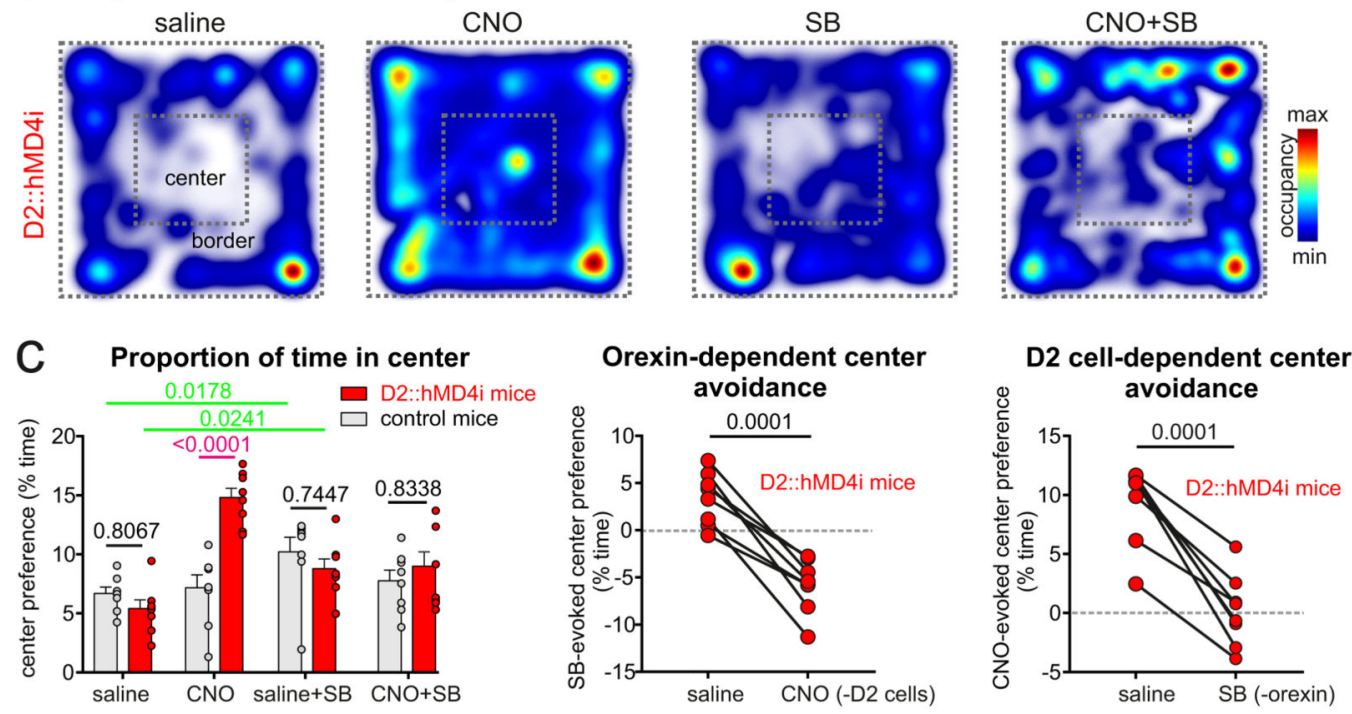

d Predator odor risk-taking

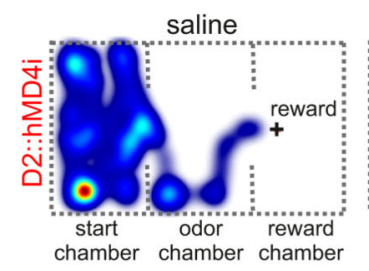

Hesitation to entry to odor chamber

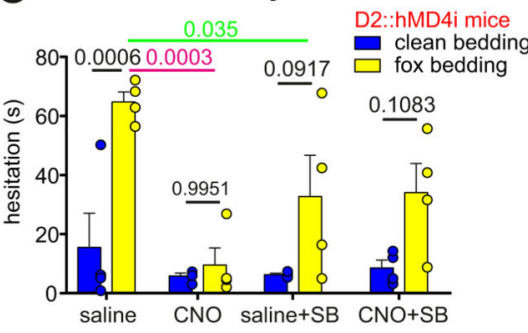

Orexin-dependent hesitation

D2 cell-dependent hesitation

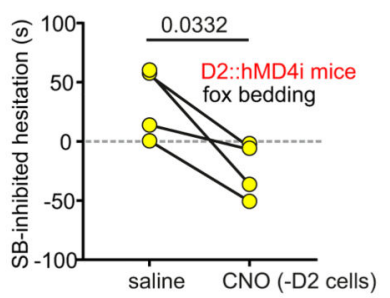

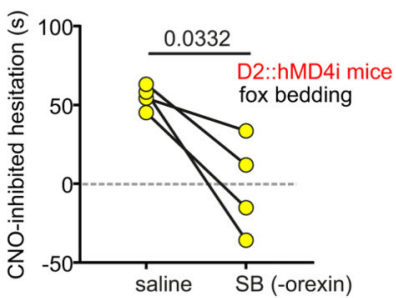

Fig. 3. Combinatorial probing of roles of $\mathrm{D} 2 \mathrm{NAc}$ and orexin cells in risk-avoidance.

A. Left: targeting scheme for hM4Di-mCherry to D2 ${ }_{\mathrm{NAc}}$ cells. Middle: localization of hM4Di-mCherry cells in NAc shell (red); representative example of $n=4$ brains. Right: Effect of CNO on a D2 ${ }_{\mathrm{NAc}}-\mathrm{hM} 4 \mathrm{Di}$ cell (representative example of $\mathrm{n}=12$ cells; mean \pm sem hyperpolarization $=3.25 \pm 0.37 \mathrm{mV}, \mathrm{p}<0.0001$ by two-tailed one-sample $\mathrm{t}$-test; $\mathrm{t}, \mathrm{df}=$ 8.687,11.

B. Examples of raw data from individual mice during a 15 min open-field test. 
C. Group data for experiment in B (values are means \pm sem and/or raw values for individual mice), numbers above data are $\mathrm{p}$ values from Sidak's or Tukey's post-tests (left plot, Twoway RM ANOVA $\mathrm{p}<0.0001 \mathrm{~F}(3,42)=13.41, \mathrm{n}=8$ mice in each group), or from two-tailed paired t-tests (middle and right plots respectively: $\mathrm{t}, \mathrm{df}=7.692,7$ and $\mathrm{t}, \mathrm{df}=7.692,7$ ). Control mice were D2::ChR2 mice.

D. Examples of raw data from individual mice in the fox odor risk-taking test.

E. Group data for experiment in $\mathrm{D}$ (values are means \pm sem or raw values for individual mice), numbers above data are p values from Sidak's or Tukey's post tests (left plot, Twoway RM ANOVA $\mathrm{p}=0.004 \mathrm{~F}(3,18)=6.348, \mathrm{n}=4$ mice in each group), or from two-tailed paired t-tests (middle and right plots respectively: $\mathrm{t}, \mathrm{df}=3.748,3$ and $\mathrm{t}, \mathrm{df}=3.748,3$ ). 